WS9-P07

\title{
Future Applications for CSEM - Shales and Monitoring
}

\author{
K.M. Strack* (KMS Technologies - KJT Enterprises Inc.)
}

\section{SUMMARY}

CSEM has become again of interest for land applications because of emerging markets such as reservoir monitoring and shale applications. They are sufficient high value to justify dealing with the complexity of a grounded dipole source, necessary to resolve both the resistors (oil reservoirs) and the conductors (host rock) equally well.

For shale application, three key applications are: Reservoir depletion, fracture monitoring and geosteering. Since increased accuracy is required at different field development stages, borehole measurements need to be added as depth and accuracy requirements increase.

For monitoring, one starts with surface only measurements. Since in more mature fields have borehole data, they should be added for a realistic 3D solution.

In all cases, feasibility studies are essential to guide the fieldwork. To estimate the risk correctly, one should acquire noise data as oilfield are likely to have pipes and corrosion protection installed. With that noise data and bounding it by the 3D seismic reservoir boundaries we can illustrate the success chance of CSEM.

Because EM responds to fluid contrast, we see more cases where EM is likely to work than not. As in these cases there is often no seismic signature it is likely to track fluid movements even better than seismic. 


\section{Amsterdam '14}

During the past 20 years, for hydrocarbon and geothermal application, controlled source electromagnetics is only used in rare instances. Recently, with the success of marine EM, it is being looked at again and major potential applications that include high value targets are emerging. These are shale / unconventionals application and reservoir monitoring where the EM response could even yield more reservoir inside than seismic data provides. At the same time technology has progressed such that we can measure and interpret today in 3D.

In an unconventional resource play (shale gas as well as shale oil) some of the key questions are: defining the reservoir, mapping of the fractures and reservoir depletion monitoring. Shale formation have an inherent string electrical anisotropy and as the hydrocarbons in shale gas or shale oil reservoirs are mostly resistive and the reservoir is relative thin, they give an anomalous electromagnetic (EM) response. The DHI effect gave rise to the entire marine EM industry and is known as Direct Hydrocarbon Indicator (DHI) or in geophysical terms the ' thin resistive layer effect' (Passalacqua, 1983; Eadie, 1980). Using modern logging tools that measure electrical anisotropy, surface tensor EM measurements can be calibrated and then become more meaningful and tie better to seismic images. In the absence of anisotropy logs, the anisotropy can be estimated using well-known equivalence principle first suggested by Keller and Frischknecht (1967).

For reservoir monitoring, time-lapse measurements as well as proper linkage to the borehole through integrating surface-to-borehole measurements is essential. Combining borehole and surface electro-magnetic measurements gives calibration points in addition to more sensitivity to fluid variations in the pore space. At the same time linking the electromagnetic (EM) information to 3D surface and borehole seismic data permits extrapolation away from the well bore. In is essential to carry out feasibility for monitoring applications because the reservoir variations will automatically make this a three-dimensional problem. This is illustrated with examples from hydrocarbon and geothermal reservoirs where even noise measurements were collected to illustrate the feasibility. The additional opportunity lies in coupling EM with seismic to get fluid movements and seal integrity.

On the hardware side the limitations are in cost and lack of interaction between transmitters and receivers, which only allow single transmitter and unfocussed receivers to be used. If we add today's accurate timing and sequencing to modern hardware we can use better arrays that allow volume focusing. This overcomes the ambiguity in the location of the subsurface response volume. Our implementation includes land sources and receivers (CSEM system), surface-to-borehole arrays and a single well system that can look tens or even $100 \mathrm{~m}$ around the wellbore and ahead of the drill bit. Extension to the marine environment is presently being built.

While modern hardware, 3D modeling and calibration can address the key challenges of land CSEM; there are still numerous remaining issues. In order to reach sufficient depth, one needs to deploy a high power transmitter, which brings operations HSE issues. In addition, grounded dipole transmitter is always sensitive to static shift caused by near electrode inhomogeneities. These need to be evaluated at every transmitter location. Given this, the effort is manageable when using the technology for high value problem such as reservoir monitoring or shale applications. 